ORIGINAL ARTICLE

\title{
Assessment and management of children aged 1-59 months presenting with wheeze, fast breathing, and/or lower chest indrawing; results of a multicentre descriptive study in Pakistan
}

\author{
T Hazir, S Qazi, Y B Nisar, S Ansari, S Maqbool, S Randhawa, Z Kundi, R Asghar, S Aslam
}

Arch Dis Child 2004;89:1049-1054. doi: 10.1136/adc.2003.035741

\begin{abstract}
Background and Aims: Using current WHO guidelines, children with wheezing are being over prescribed antibiotics and bronchodilators are underutilised. To improve the WHO case management guidelines, more data is needed about the clinical outcome in children with wheezing/pneumonia overlap.

Methodology: In a multicentre prospective study, children aged 1-59 months with auscultatory/audible wheeze and fast breathing and/or lower chest indrawing were screened. Response to up to three cycles of inhaled salbutamol was recorded. The responders were enrolled and sent home on inhaled bronchodilators, and followed up on days 3 and 5 .

Results: A total of 1622 children with wheeze were screened from May 2001 to April 2002, of which 1004 (61.8\%) had WHO defined non-severe and 618 (38.2\%) severe pneumonia. Wheeze was audible in only $595(36.7 \%)$ of children. Of 1004 non-severe pneumonia children, 621 (61.8\%) responded to up to three cycles of bronchodilator. Of 618 severe pneumonia children, only $166(26.8 \%)$ responded. Among responders, $93(14.9 \%)$ in the non-severe and $63(37.9 \%)$ children in the severe pneumonia group showed subsequent deterioration on follow ups. No family history of wheeze, temperature $>100^{\circ} \mathrm{F}$, and lower chest indrawing were identified as predictors of subsequent deterioration.

Conclusions: Two third of children with wheeze are not identified by current WHO ARI (acute respiratory infections) guidelines. Antibiotics are over prescribed and bronchodilators under utilised in children with wheeze. Children with wheeze constitute a special ARI group requiring a separate management algorithm. In countries where wheeze is common it would be worthwhile to train health workers in use of the stethoscope to identify wheeze.
\end{abstract}

See end of article for
authors' affiliations

Correspondence to: Dr T Hazir, Associate Professor, The Children's Hospital, Islamabad, Pakistan; arichi99@ hotmail.com

Accepted 2 February 2004
$\mathrm{T}$ he World Health Organisation (WHO) has developed an acute respiratory infections (ARI) case management strategy that employs simple clinical signs to diagnose pneumonia, followed by empirical antimicrobial treatment. The main objective of the ARI programme is to reduce pneumonia mortality in developing countries. Due to this overwhelming concern all pneumonia is presumed to be bacterial and treated with an antibiotic. There is a concern that a large number of children are getting antibiotics unnecessarily since they may have viral pneumonia. There are difficulties in distinguishing viral from bacterial pneumonia; even radiology seems to be of little help..$^{2-5}$ Wheeze is more commonly associated with a viral infection, especially respiratory syncytial virus (RSV). ${ }^{67}$ Children with wheeze can have fast breathing and lower chest indrawing, which can place them in the category of non-severe and severe pneumonia according to the current ARI case management guidelines. In order to address the issue of over categorisation of pneumonia and rationalise the use of antibiotics in children with wheeze, the current WHO ARI case management guidelines recommend giving two cycles of rapid acting inhaled bronchodilator at 15 minute intervals to children with audible wheeze and fast breathing and/or lower chest indrawing before reassessment for antibiotic prescription. ${ }^{8}$ If the fast breathing and/or lower chest indrawing disappears after up to two doses, they are classified as "no pneumonia" and sent home on oral bronchodilators. These children are not routinely followed up and little is known about their clinical course. As far as we know these guidelines have not been formally evaluated. The current generic WHO integrated management of childhood illness (IMCI) guidelines do not include management of wheeze, ${ }^{9}$ whereas the IMCI guidelines adapted in Pakistan recommend one dose of fast acting bronchodilator and reassessment after 30 minutes. ${ }^{10}$

There are certain concerns regarding the current ARI case management guidelines for wheeze in children. In order for a child to enter a management algorithm for wheeze, he/she must have an audible wheeze. It has been documented that audible wheeze is present in only $30 \%$ of the children who have auscultatory wheeze. ${ }^{11}$ Therefore, the majority of children with wheeze are not being identified by health workers. A large proportion of children with wheeze are, therefore, being diagnosed as having pneumonia and given antibiotics unnecessarily.

The data regarding the clinical profile and subsequent clinical course of children who are sent home on oral bronchodilators after responding to up to two cycles of inhaled bronchodilators have not been well documented. Some questions arise with regard to these children: (1) What proportion will deteriorate subsequently, and is that significant enough to require a routine follow up at 48 hours for reassessment? (2) Can any clinical predictors identify the subgroup at higher risk of deteriorating? To answer the above questions, we followed up wheezing children for 5-7 days

Abbreviations: $A R I$, acute respiratory infection; $I M C l$, integrated management of childhood illness; IQR, interquartile range; $M D I$, metered dose inhaler; OPD, outpatient department; RSV, respiratory syncytial virus; WHO, World Health Organisation 
who were reassessed as "no pneumonia with wheeze" after responding to a trial of rapid acting bronchodilators.

\section{METHODS}

\section{Study population and site}

This study was prospectively conducted in the outpatient departments (OPD) of Rawalpindi General Hospital, Rawalpindi, The Children's Hospital, Islamabad, and The Children's Hospital, Lahore, in Pakistan (fig 1). Study physician screened children 1-59 months of age presenting with cough and/or difficult breathing for both audible and auscultatory wheeze using the WHO ARI guidelines. ${ }^{8}$ The patients with wheeze and fast breathing were given inhaled salbutamol and reassessed after up to three cycles of bronchodilator therapy, repeated (if necessary) at 15 minute interval. To overcome the electrostatic discharges created in the volume spacer, four actuations of inhaled salbutamol (Ventolin inhaler $100 \mu \mathrm{g} /$ actuation, Glaxo Smithkline \& Beecham) were used by metered dose inhaler (MDI) before the first inhalation and two actuations before the subsequent two inhalations. Children were enrolled if audible or auscultatory wheeze and their fast breathing and/or lower chest indrawing had disappeared after responding to inhaled bronchodilators. A child was excluded if any WHO defined danger signs were present, if he/she had received adequate antibiotic treatment in the past 48 hours, if there was complicating acute non-pulmonary or chronic illness, if the child could not be followed up (that is, lived outside the municipal limits of the city), if their parents/guardians refused to give consent, and if they had been previously enrolled in this study.

All enrolled patients were advised to have two actuations of inhaled salbutamol using MDI and spacer device at eight hourly intervals at home. MDI and spacer device
(Volumhaler, Maxwell Mabrose) with face mask (Pulmimask, Barrett Hodgson) use was demonstrated to the mother after enrolment and was repeated by her in front of the study staff. Each enrolled child was followed up at day 3 and day 5. The children who did not come for follow up were followed up at home within 24 hours of the follow up day (fig 1).

The purpose of the study was explained to the parents, and an oral witnessed informed consent in their local language was obtained for all patients for screening and enrolment. The study was approved by the institutional ethical review boards of the Pakistan Institute of Medical Sciences, Islamabad, Pakistan, and the Secretariat Committee on Research Involving Human Subjects, WHO, Geneva.

\section{Sample size}

Using a standard formula for single proportion, ${ }^{12}$ and assuming $50 \%$ prevalence of wheeze in children with ARI, and a power of $80 \%$ with $95 \%$ confidence level, the sample size for descriptive analysis was calculated to be 384 . We were also interested in the treatment outcomes. Since the outcome was unknown, taking a conservative proportion of $\mathrm{p}=0.5$ of children responding to treatment gave us the largest sample size possible of 1600 children to study the outcomes.

\section{Health personnel}

The study physicians were trained in standard ARI case management, with emphasis on recognition and management of wheeze and filling of case report forms. After enrolment, children who deteriorated subsequently received antibiotic and/or steroid therapy as directed by their physician. Children whose parents withdrew consent were treated according to the standard hospital treatment guidelines.

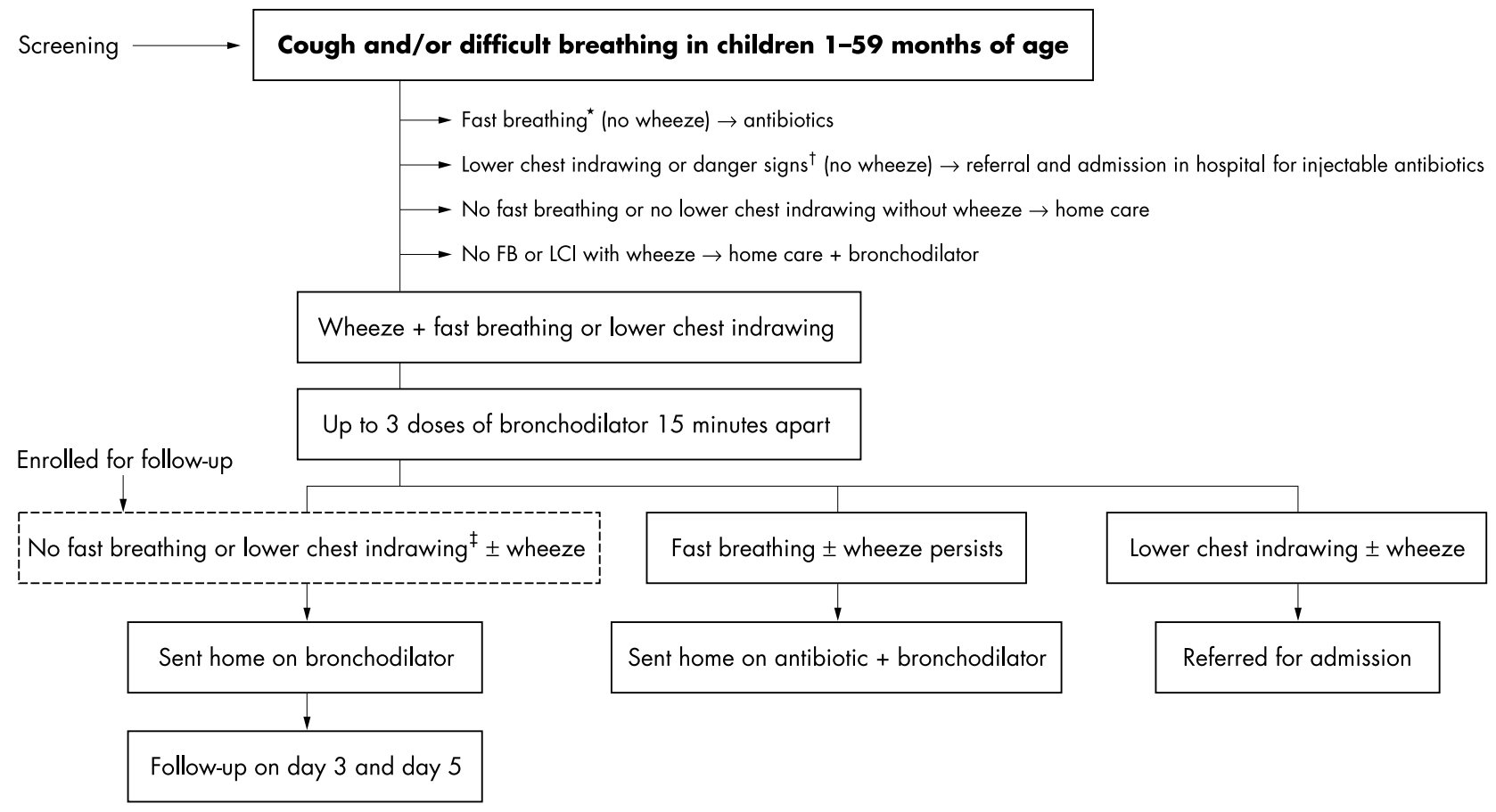

Figure 1 Flow chart of the study. ${ }^{*}$ Children with fast breathing were categorised as non-severe pneumonia. Fast breathing is defined as $\geqslant 60$ breaths per minute for up to 2 months of age, $\geqslant 50$ breaths per minute for $2-11$ months of age, and $\geqslant 40$ breaths per minute for $12-59$ months of age. tChildren with lower chest indrawing were categorised as severe pneumonia, whereas those with danger signs (inability to drink, convulsions, vomiting everything, lethargy or unconscious, severe malnutrition) were categorised as very severe disease. $\ddagger$ These patients were enrolled and followed up at day 3 and day 5 . Day of enrolment was considered as day 0 . Information on all the patients who presented with wheeze and were screened for the study, whether they were enrolled or not, were recorded by filling in the screening form. These data were also analysed. 


\section{Outcome measures}

The final outcome was deterioration or completely well on day 5-7. Subsequent deterioration was defined as: (1) his/her respiratory rate failed to return to a level below the age specific cut off after up to three doses of inhaled bronchodilators at any follow up; (2) he/she developed any WHO defined danger sign at any stage; (3) he/she developed life threatening or serious adverse drug reaction; or (4) he/she received any antibiotic during the first 48 hours after enrolment or required steroids subsequent to enrolment

\section{Data analysis}

Data were entered and validated twice using Epi Info 6.04d (software for health statistics). Demographic indicators and clinical features recorded at enrolment, day 3, and day 5 were analysed. The initial response to inhaled bronchodilator therapy was analysed. The $\chi^{2}$ test was used for categorical variables and the $t$ test was used for continuous variables. For categorical variables the analysis included estimates of odds ratio and $95 \%$ confidence intervals by univariate analysis. For the $t$ test only $\mathrm{p}$ values were reported in the results. A multivariate model was constructed using forward stepwise logistic regression to identify determinants of subsequent deterioration.

\section{RESULTS}

From May 2001 to April 2002, we screened 1628 children with fast breathing and/or lower chest indrawing along with auscultatory wheeze. Six patients who were lost to follow up were excluded; data were analysed for 1622 children.

\section{Screening}

At screening, 1004 (61.8\%) were classified as having nonsevere pneumonia and $618(38.2 \%)$ severe pneumonia with wheeze. Audible wheeze was present in only 595 (36.7\%) children. All children were then given up to three cycles of inhaled bronchodilators at 15 minute intervals, reassessed, and then classified as having either pneumonia or no pneumonia.

Of 621 responders in the non-severe pneumonia group, 268 $(43.2 \%)$ responded to the first cycle, $262(42.1 \%)$ to the second, and $91(14.7 \%)$ to the third cycle. Of 166 responders in the severe pneumonia group, $34(20.1 \%)$ responded to the first cycle, $39(23.1 \%)$ to the second, and $96(56.8 \%)$ to the third cycle.

Table 1 summarises baseline characteristics of responders and non-responders to inhaled bronchodilators at the time of screening.

\section{Enrolment and follow up}

Children in whom fast breathing and lower chest indrawing disappeared after the bronchodilator trial at screening were enrolled for further follow up. Of 621 children enrolled from the non-severe pneumonia group, $548(88.2 \%)$ were well on day 3 and required no further treatment; $528(96.3 \%)$ of these continued to be completely well on day 5 follow up.

Of 166 children enrolled from the severe pneumonia group, $110(66.2 \%)$ were well on day 3 follow up and $103(96.3 \%)$ continued to be well on day 5 follow up. Children who deteriorated were referred for further assessment and management. Children with severe pneumonia who responded to the third cycle did not have a greater incidence of deterioration on subsequent follow ups when compared with those who responded to the first two cycles (data not shown).

Table 2 summarises baseline characteristics of enrolled patients by clinical outcome on day 5 .

We analysed various clinical signs and symptoms in an attempt to identify those which could serve as possible predictors of subsequent deterioration. These signs can be used to classify high risk children who can be monitored closely. This would help improve the existing ARI case management guidelines. We constructed a forward stepwise logistic regression model including the variables identified as

Table 1 Comparison of baseline characteristics of responders and non-responders to inhaled bronchodilators at the time of screening $(n=1622)$

\begin{tabular}{|c|c|c|c|}
\hline Variables & Responders ( $n=787$ ) & Non-responders $(n=835$ ) & OR $(95 \% \mathrm{Cl})^{*}, \mathrm{p}$ value \\
\hline \multicolumn{4}{|l|}{ Demographic indicators } \\
\hline Male & $534(67.9 \%)$ & $534(64.0 \%)$ & $0.84(0.68$ to 1.04$), 0.11$ \\
\hline Age 1-11 months & $385(48.9 \%)$ & $424(50.8 \%)$ & 1.08 (0.88 to 1.32$), 0.48$ \\
\hline Median (IQR) & $6.0(4.0-8.0)$ & $5.0(4.0-8.0)$ & \\
\hline Age $12-59$ months & $402(51.1 \%)$ & $411(49.2 \%)$ & \\
\hline Median (IQR) & $25.5(18.0-37.0)$ & $22.0(15.0-34.0)$ & \\
\hline \multicolumn{4}{|l|}{ History and signs } \\
\hline Fever & $343(43.6 \%)$ & $691(82.8 \%)$ & $6.48(5.11$ to 8.23$),<0.00001$ \\
\hline Difficult breathing & $766(97.3 \%)$ & $824(98.7 \%)$ & $2.46(0.95$ to 6.62$), 0.06$ \\
\hline \multicolumn{4}{|l|}{ Duration of illness } \\
\hline Up to 2 days & $465(59.1 \%)$ & $437(52.3 \%)$ & \\
\hline Median (IQR) & $2.0(1.0-2.0)$ & $2.0(1.0-2.0)$ & \\
\hline More than 2 days & $322(40.9 \%)$ & $398(47.7 \%)$ & $1.32(1.08$ to 1.61$), 0.01$ \\
\hline Median (IQR) & $3.0(3.0-4.0)$ & $3.0(3.0-5.0)$ & \\
\hline No past history of wheezing episode & $331(42.1 \%)$ & $381(45.6 \%)$ & $1.16(0.95$ to 1.41$), 0.16$ \\
\hline No family history of wheeze & 459 (58.3\%) & $506(60.6 \%)$ & $1.10(0.90$ to 1.35$), 0.36$ \\
\hline Presence of audible wheeze & $205(26.0 \%)$ & $390(46.7 \%)$ & 2.49 (2.01 to 3.08$),<0.00001$ \\
\hline \multicolumn{4}{|l|}{ Actual temperature (in $\left.{ }^{\circ} \mathrm{F}\right)$} \\
\hline Up to $100^{\circ} \mathrm{F}$ & $762(96.8 \%)$ & $689(82.5 \%)$ & \\
\hline Median (IQR) & $98.6(98.6-98.6)$ & $99.0(98.6-99.0)$ & \\
\hline More than $100^{\circ} \mathrm{F}$ & $25(3.2 \%)$ & $146(17.5 \%)$ & $6.46(4.10$ to 10.2$),<0.00001$ \\
\hline Median (IQR) & $100.8(100.4-101.7)$ & $101.0(100.2-101.2)$ & \\
\hline \multicolumn{4}{|l|}{ Weight for age $Z$ score } \\
\hline Median (IQR) & $-0.95(-1.6$ to -0.22$)$ & $-0.95(-1.7$ to -0.18$)$ & 0.69 \\
\hline Height for age $\mathrm{Z}$ score & & & \\
\hline Median (IQR) & $-0.52(-1.3$ to 0.16$)$ & $-0.66(-1.4$ to 8.0$)$ & 0.38 \\
\hline \multicolumn{4}{|l|}{ ARI classification } \\
\hline Non-severe pneumonia with wheeze & $621(78.9 \%)$ & $383(45.9 \%)$ & \\
\hline $\begin{array}{l}\text { Severe pneumonia (lower chest } \\
\text { indrawing) with wheeze }\end{array}$ & $166(21.1 \%)$ & $452(54.1 \%)$ & $4.41(3.53$ to 5.53$),<0.0001$ \\
\hline
\end{tabular}


significant by univariate analysis. Multiple logistic regression identified history of fever, presence of audible wheeze, actual temperature more than $100^{\circ} \mathrm{F}$, and lower chest indrawing as independent predictors of poor response to bronchodilator at the time of screening (table 3 ). Temperature $>100^{\circ} \mathrm{F}$, lower chest indrawing, and no family history of wheeze were identified as predictors of subsequent deterioration (table 3 ).

The risk factors associated with non-response at screening and subsequent deterioration after enrolment were further studied for their sensitivity, specificity, and positive predictive value. History of fever was found to be the most sensitive predictor $(82.7 \%)$ of poor response to bronchodilator, although its specificity was only $56.4 \%$. On the other hand a temperature of $>100^{\circ} \mathrm{F}$ was highly specific for poor response to bronchodilator and subsequent deterioration, but had very low sensitivity. The overall positive predictive value was best for a temperature of $>100^{\circ} \mathrm{F}$ (table 4 ).

No deaths were reported in children with non-severe or severe pneumonia and wheeze in which antibiotics were withheld.

\section{DISCUSSION}

Our data have both global and country specific implications and we will address both. The current WHO ARI case management guidelines do not recommend the use of a stethoscope by the health workers for obvious reasons such as training, costs, and logistics. One of the major concerns regarding management of children with wheeze is the identification of all wheezy children by the health personnel. Our results show that only one third of the children with auscultatory wheeze will have audible wheeze; thus a large majority of children with wheeze will not be identified by the health workers using only WHO ARI guidelines. Hence, the majority of the children with wheeze, fast breathing, and/or lower chest indrawing will be classified as having pneumonia. Our results show that children with auscultatory wheeze responded better to a trial of inhaled bronchodilator compared to children having audible wheeze, and also had a better treatment outcome when sent home without an antibiotic. Nearly one third of the children with auscultatory wheeze had lower chest indrawing, who would otherwise be classified as having "severe pneumonia" and be treated with injectable antibiotics. Our data also showed that the sign of lower chest indrawing, which is used for classifying children as having "severe pneumonia", disappeared in one third of these children after up to three cycles of inhaled bronchodilator. The majority of these children continue to do well without antibiotics on follow up. If health workers are trained in auscultation, a great majority of children with wheeze may not receive antibiotics.

There are considerable potential benefits to improving the management of children with wheeze. These include better management of wheezing children, reduction in antibiotic use leading to potential reduction in antimicrobial resistance, reduction in referral and admissions, reduction in injectable use resulting in less risk of hepatitis B and HIV, reduced risk of nosocomial infections, potential reduction in cost of therapy and hospitalisation, and less pressure on limited hospital beds. In countries where wheeze is commonly prevalent, it will be worthwhile to teach health workers how to use the stethoscope in order to reap all these benefits.

The current WHO ARI guidelines, based on clinical experience, recommend two cycles of rapid acting bronchodilator given at a 15 minute interval to the children who present with wheeze and fast breathing and/or lower chest indrawing. Our results provide scientific data in support of this recommendation. Only a small proportion of children in the non-severe pneumonia category benefit from the third cycle; they have a higher risk of persistent wheezing and signs of deterioration on subsequent follow up. Our results also support the recommendation of a 15 minute gap between the two cycles of bronchodilator.

Table 2 Comparison of baseline characteristics by clinical outcome on day 5 (enrolled patients, $n=787^{*}$ )

\begin{tabular}{|c|c|c|c|}
\hline Variables & Well $(n=631)$ & Deterioration $(n=156)$ & OR $(95 \% \mathrm{Cl}) \dagger, \mathrm{p}$ value \\
\hline \multicolumn{4}{|l|}{ Demographic indicators } \\
\hline Male & $435(68.9 \%)$ & 99 (63.5\%) & $0.78(0.53$ to 1.15$), 0.22$ \\
\hline Age $1-11$ months & $290(46.0 \%)$ & $95(60.9 \%)$ & 1.83 (1.26 to 2.66$), 0.01$ \\
\hline Median (IQR) & $6.0(4.0-8.0)$ & $5.0(3.0-8.0)$ & \\
\hline Age $12-59$ months & $341(54.0 \%)$ & $61(39.1 \%)$ & \\
\hline Median (IQR) & $28.0(18.0-38.5)$ & $21.0(15.5-34.5)$ & \\
\hline \multicolumn{4}{|l|}{ History and signs } \\
\hline Fever & $252(39.9 \%)$ & $91(58.3 \%)$ & 2.11 (1.44 to 3.03$),<0.0001$ \\
\hline Difficult breathing & 612 (97.0\%) & $154(98.8 \%)$ & $3.77(0.52$ to 77.21$), 0.29$ \\
\hline \multicolumn{4}{|l|}{ Duration of illness } \\
\hline Up to 2 days & $387(61.3 \%)$ & $78(50.0 \%)$ & \\
\hline Median (IQR) & $2.0(1.0-2.0)$ & $2.0(1.0-2.0)$ & \\
\hline More than 2 days & $244(38.7 \%)$ & $78(50.0 \%)$ & $1.59(1.10$ to 2.29$), 0.01$ \\
\hline Median (IQR) & $3.0(3.0-5.0)$ & $3.0(3.0-4.2)$ & \\
\hline No past history of wheezing episode & $247(39.1 \%)$ & $84(53.8 \%)$ & 1.81 (1.29 to 2.62$), 0.01$ \\
\hline No family history of wheeze & $348(55.2 \%)$ & $111(71.2 \%)$ & $2.02(1.36$ to 3.02$), 0.01$ \\
\hline Presence of audible wheeze & 151 (23.9\%) & $54(34.6 \%)$ & 1.68 (1.13 to 2.50$), 0.01$ \\
\hline \multicolumn{4}{|l|}{ Actual temperature (in ${ }^{\circ} \mathrm{F}$ ) } \\
\hline Up to $100^{\circ} \mathrm{F}$ & $621(98.4 \%)$ & $141(90.4 \%)$ & \\
\hline Median (IQ Range) & $98.6(98.6-98.6)$ & $98.6(98.6-99.0)$ & \\
\hline More than $100^{\circ} \mathrm{F}$ & $10(1.6 \%)$ & $15(9.6 \%)$ & $6.61(2.73$ to 16.11$),<0.0001$ \\
\hline Median (IQR) & $101.5(100.3-102.3)$ & $100.8(100.4-101.0)$ & \\
\hline \multicolumn{4}{|l|}{ Weight for age $Z$ score } \\
\hline Median (IQR) & $-0.99(-1.6$ to -0.24$)$ & $-0.72(-1.5$ to -3.75$)$ & 0.16 \\
\hline Height for age $\mathrm{Z}$ score & & & \\
\hline Median (IQR) & $-0.52(-1.4$ to 0.13$)$ & $-0.57(-1.2$ to 0.21$)$ & 0.49 \\
\hline \multicolumn{4}{|l|}{ ARI classification } \\
\hline Non-severe pneumonia with wheeze & $528(83.6 \%)$ & $93(59.6 \%)$ & \\
\hline $\begin{array}{l}\text { Severe pneumonia (lower chest indrawing) } \\
\text { with wheeze }\end{array}$ & $103(16.4 \%)$ & $63(40.4 \%)$ & $3.47(2.32$ to 5.19$),<0.0001$ \\
\hline
\end{tabular}


Table 3 Predictors identified by multivariate logistic regression* of non-response to inhaled bronchodilator at the time of screening and subsequent deterioration until day 5 after enrolment

\begin{tabular}{|c|c|c|}
\hline & OR $(95 \% \mathrm{Cl})$ & p value \\
\hline \multicolumn{3}{|l|}{$\begin{array}{l}\text { Non-response to inhaled bronchodilator at the time of screening } \\
\text { Screened patients }(n=1622)\end{array}$} \\
\hline History of fever & 4.06 (3.18 to 5.18$)$ & $<0.00001$ \\
\hline Presence of audible wheeze & $1.79(1.41$ to 2.26$)$ & $<0.00001$ \\
\hline Temperature more than $100^{\circ} \mathrm{F} \dagger$ & 2.99 (1.89 to 4.74$)$ & $<0.00001$ \\
\hline Lower chest indrawing (severe pneumonia) with wheeze & $2.47(1.94$ to 3.14$)$ & $<0.00001$ \\
\hline \multicolumn{3}{|l|}{$\begin{array}{l}\text { Subsequent deterioration until day } 5 \text { after enrolment } \\
\text { Enrolled patients }(n=787)\end{array}$} \\
\hline No family history of wheeze & $1.76(1.19$ to 2.62$)$ & 0.005 \\
\hline Temperature more than $100^{\circ} \mathrm{F} \dagger$ & $3.94(1.66$ to 9.31$)$ & 0.002 \\
\hline Lower chest indrawing (severe pneumonia) with wheeze & $2.96(1.99$ to 4.40$)$ & $<0.00001$ \\
\hline \multicolumn{3}{|c|}{$\begin{array}{l}\text { *For modelling purpose continuous variables such as age, temperature, and duration of illness were recoded in to } \\
\text { categorical variables. } \\
\text { †Against temperature } \leqslant 100^{\circ} \mathrm{F} \text {. }\end{array}$} \\
\hline
\end{tabular}

Our data also show that only two thirds of children with fast breathing and one fifth of children with chest indrawing and wheeze will respond to a single cycle of bronchodilator. Thus, our data do not support the current IMCI guidelines adaptation for Pakistan, which recommend a single cycle of inhaled bronchodilator followed by an assessment 30 minutes later. They probably result in a lot more children with wheeze being prescribed antibiotics unnecessarily. Furthermore, our data show that the response to inhaled bronchodilators becomes apparent within 15 minutes; the waiting for 30 minutes before reassessment also needs to be modified. Modifying Pakistani IMCI guidelines for wheezing children will potentially reduce antibiotic use and save an additional 15 minutes of waiting at the health facility for many patients.

Our results show that in children with lower chest indrawing and wheeze, the maximum response $(56.6 \%)$ is seen after the third cycle of inhaled bronchodilators. Therefore, children with severe pneumonia and wheeze constitute a special group where a third cycle of bronchodilators may be justified before a decision about their referral is made. The current recommendation of not withholding of antibiotics in children who still have lower chest indrawing after two doses of inhaled bronchodilator is based on the concern that it may not be safe. According to our results, a great majority $(66.2 \%)$ of the children in whom lower chest indrawing disappears after the third cycle, will continue to show improvement and will not deteriorate without an antibiotic. There was no death in this group of children, even when antibiotics were withheld from them. If data from other parts of the world show similar results, there would be a need to revise the current ARI guidelines regarding the number of inhaled bronchodilator cycles in children with lower chest indrawing and wheeze.

Our data show that the majority of children who deteriorate subsequently do so in the first 48 hours. The current ARI guidelines do not recommend a routine follow up at 48 hours for the children with wheeze. Based on our data, a 48 hour follow up may be required for all wheezy children irrespective of their response to bronchodilator at the time of presentation. Our data have shown that there are certain clinical signs which have a significant association with the risk of subsequent deterioration. We evaluated the possible usefulness of these signs in identifying high risk children. A temperature of $>100^{\circ} \mathrm{F}$ had the best overall positive predictive value followed by the presence of lower chest indrawing. Children having these clinical signs can be classified as a high risk group. Carers of these children should be counselled in greater detail. There should be a greater emphasis on teaching the carers of these children the signs of deterioration so that they can bring these children to the health facility in time.

To summarise, children with wheeze constitute a special ARI group. Using current WHO ARI case management guidelines, the majority of the children with bronchospasm are not being identified. These children are getting antibiotics unnecessarily. Bronchodilators are being under utilised. There is a need to develop a separate management algorithm

Table 4 Sensitivity, specificity, and positive predictive value of risk factors associated with non-response to inhaled bronchodilator at the time of screening, and subsequent deterioration until day 5 after enrolment

\begin{tabular}{|c|c|c|c|}
\hline Sign & Sensitivity (\%) & Specificity (\%) & $\begin{array}{l}\text { Positive predictive } \\
\text { value (\%) }\end{array}$ \\
\hline \multicolumn{4}{|l|}{ Risk factors at screening } \\
\hline History of fever & 82.7 & 56.4 & 66.8 \\
\hline Presence of audible wheeze & 46.7 & 73.9 & 65.5 \\
\hline \multirow{2}{*}{$\begin{array}{l}\text { Temperature more than } 100^{\circ} \mathrm{F} \\
\text { Lower chest indrawing (severe } \\
\text { pneumonia) with wheeze }\end{array}$} & 17.4 & 96.8 & 85.5 \\
\hline & 54.1 & 78.9 & 73.0 \\
\hline \multicolumn{4}{|l|}{ Risk factors after enrolment } \\
\hline No family history of wheeze & 71.1 & 37.7 & 24.1 \\
\hline \multirow{2}{*}{$\begin{array}{l}\text { Temperature more than } 100^{\circ} \mathrm{F} \\
\text { Lower chest indrawing (severe } \\
\text { pneumonia) with wheeze }\end{array}$} & 9.6 & 98.4 & 60.0 \\
\hline & 40.3 & 83.6 & 37.9 \\
\hline
\end{tabular}


for wheeze. In order to improve ARI case management, health workers should be trained to use the stethoscope. All children with wheeze must be followed up at 48 hours.

There are vast gaps in the current knowledge of children with wheeze, especially in the developing countries. There is a need to collect more data on children with wheeze before a separate algorithm for management of wheeze is developed. Identical data are currently being collected in Colombia, Egypt, Ghana, and Thailand that will enable us to understand this problem better.

\section{Authors' affiliations}

T Hazir, Y B Nisar, S Ansari, The Children's Hospital, Islamabad, Pakistan

S Qazi, World Health Organisation, Geneva, Switzerland

S Maqbool, S Randhawa, The Children's Hospital, Lahore, Pakistan

Z Kundi, R Asghar, S Aslam, Rawalpindi General Hospital, Rawalpindi, Pakistan

The Department of Child and Adolescent Health and Development, WHO, Geneva funded the study

\section{REFERENCES}

1 World Health Organisation. The management of acute respiratory infections in children. Practical guidelines for outpatient care. Geneva: World Health Organisation, 1995.
2 Putto A, Ruuskanen O, Meurman O. Fever in respiratory virus infections. Am J Dis Child 1986;140:1159-63.

3 McCarthy PL, Spiesel SZ, Stashwick CA, et al. Radiographic findings and etiologic diagnosis in ambulatory childhood pneumonias. Clin Pediatr 1981:20:686-91.

4 Bettenay FA, de Campo JF, McCrossin DB. Differentiating bacterial from viral pneumonias in children. Pediatr Radiol 1988;18:453-4.

5 Courtoy I, Lande AE, Turner RB. Accuracy of radiographic differentiation of bacterial from non-bacterial pneumonia. Clin Pediatr (Phila) 1989;28:261-4.

6 Panitch HB. Bronchiolitis in infants. Curr Opin Pediatr $2001 ; 13: 256-60$.

7 Turner RB, Lande AE, Chase $\mathrm{P}$, et al. Pneumonia in pediatric outpatients: cause and clinical manifestations. J Pediatr 1987;111:194-200.

8 World Health Organisation. Acute respiratory infections in children. Case management in small hospitals in developing countries. A manual for doctors and other senior health workers. Programme for the Control of Acute Respiratory Infections. WHO/ARI/90.5. Geneva: World Health Organisation, 1990

9 World Health Organisation. Handbook of $\mathrm{IMCl}$ integrated management of childhood illness. WHO/FCH/CAH/00.12. Geneva: Department of Child and Adolescent Health and Development, World Health Organisation and UNICEF, 2000.

10 Integrated Management of Childhood Illness (IMCI). Treat the child. WHO/ PAK/00-2.D. Ministry of Health, Government of Pakistan, World Health Organisation, and UNICEF, October 2000

11 Sachdev HPS, Vasanthi B, Satyanarayana L, et al. Simple predictors to differentiate acute asthma from ARI in children: implications for refining case management in the ARI Control Programme. Indian Pediatr 1995;31:1251-9.

12 Lwanga SK, Lemishow S. Sample size determination in health studies. Geneva: World Health Organisation, 1991:25.
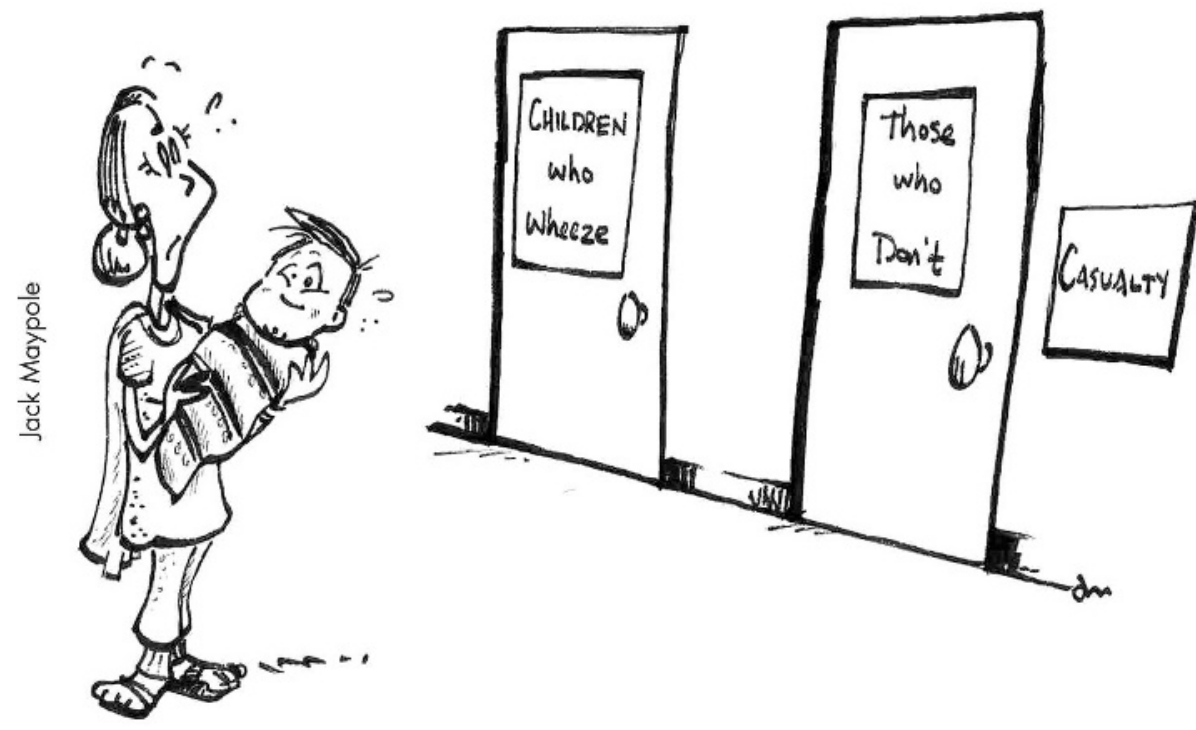